\title{
Effects of inclusion size and location on very-high-cycle fatigue behavior for high strength steels
}

\author{
Zhengqiang Lei, Youshi Hong*, Jijia Xie, Chengqi Sun, Aiguo Zhao \\ State Key Laboratory of Nonlinear Mechanics, Institute of Mechanics, Chinese Academy of Sciences, Beijing 100190, PR China
}

\section{A R T I C L E I N F O}

\section{Article history:}

Received 14 June 2012

Accepted 30 July 2012

Available online 15 August 2012

\section{Keywords:}

Very-high-cycle fatigue

FGA

Inclusion

High strength steels

\begin{abstract}
A B S T R A C T
Fatigue tests with rotary bending $(52.5 \mathrm{~Hz})$ and ultrasonic cycling $(20 \mathrm{kHz})$ methods were performed, respectively on two high strength steels so as to investigate the effects of inclusion size and location on the fatigue behavior from low cycle fatigue to very-high-cycle fatigue (VHCF) regimes. Under rotary bending test, four fracture patterns were observed with respect to different locations of inclusion on fracture surface. The mean crack growth rate within FGA (fine granular area of crack origin) was estimated to give the value with the magnitude of $10^{-13} \mathrm{~m} / \mathrm{cycle}$ for the specimens with the fracture pattern of "FGA + surface fisheye". Under ultrasonic cycling test, the size effect of inclusions on VHCF was investigated with two groups of specimens. One is with the inclusion size between 20 and $60 \mu \mathrm{m}$ and the other is with the inclusion size between 10 and $30 \mu \mathrm{m}$. The results show that large inclusions lowered the proportion of net FGA at given stress levels and led to the shortening of VHCF life. The degradation of VHCF strength caused by the increase of inclusion size is ascribed to the decrease of the critical stress of FGA formation for large inclusions.
\end{abstract}

(c) 2012 Elsevier B.V. All rights reserved.

\section{Introduction}

It is well known that $S-N$ curve of ferrous materials tends to a limit between $10^{6}$ and $10^{7}$ cycles of loading. This fatigue limit was not challenged until the fatigue failure beyond $10^{7}$ cycles was first reported in 1980s [1,2]. Therefore, very-high-cycle fatigue (VHCF) is named as the fatigue failure beyond $10^{7}$ cycles, which has been confirmed to be existed in various types of materials [3,4]. This suggests that the conventional fatigue limit at $10^{7}$ cannot provide safety design data for engineering structures. In fact, there is a need for many engineering structures to endure long period of cyclic loading up to $10^{10}$ cycles, such as aircrafts, automobiles, ship, railway, etc. [5]. Obviously, it is an important topic to clarify the behavior and mechanism of VHCF failures.

For high strength steels, the process of interior crack initiation and early growth induced by inclusions is the typical feature of VHCF failures. Around the inclusion, a characteristic rough region with fine granular area is presented, and this rough region is always surrounded by a flat region thus to form a whole "fisheye" morphology [6-8]. Due to the related observation approach, this rough area is named as optically dark area (ODA [9]), granular bright facet (GBF [10]), or fine granular area (FGA [11]) in

\footnotetext{
* Corresponding author. Tel.: +86 10 82543966; fax: +86 1062561284 .

E-mail address: hongys@imech.ac.cn (Y. Hong).
}

literature. In this paper, the name of FGA is used for this rough region.

The formation of FGA corresponds to the period of VHCF crack initiation, which consumes the majority of the fatigue life. Thus several mechanisms have been proposed to explain the formation of FGA, such as "hydrogen embrittlement-assisted cracking" [12], "dispersive decohesion of spherical carbide" [13], and "polygonization and debonding" [11]. Each of these explanations is reasonable in certain case, but the influential factors and the related mechanism of FGA have not been fully understood yet.

As the most common defect for the fatigue failures of high strength steels, the inclusion of the materials has been paid close attention to and its effect has been included in the models for fatigue life and strength prediction [14-16]. These models are empirical formulae based on the experimental results, although the mechanism of the influence of inclusions on fatigue failures is not clear. In other words, there is still a lack of knowledge in the interpretation of the influential tendency and mechanism of inclusions on the fatigue behavior.

In this paper, an experimental investigation is carried out for the effects of inclusion size and location on the fatigue behavior from low cycle fatigue to VHCF regime via fatigue tests of rotary bending $(52.5 \mathrm{~Hz})$ and ultrasonic cycling $(20 \mathrm{kHz})$. The fractography of broken specimens subjected to rotary bending test was examined to reveal that fatigue fracture pattern transition caused by inclusion location. Under ultrasonic loading, the size effect of inclusions on VHCF was investigated. The mechanism of the 
influence of inclusions on fatigue life and fatigue strength is discussed, and the mean crack growth rate in FGA is estimated.

\section{Experimental procedure}

\subsection{Materials and specimens}

The test materials in the present investigation are two high carbon chromium steels. One material is with large inclusions (designated as $L$ ) and the chemical compositions (mass percentage) are: $1.06 \mathrm{C}, 1.04 \mathrm{Cr}, 0.88 \mathrm{Mn}, 0.34 \mathrm{Si}, 0.027 \mathrm{P}$, and $0.005 \mathrm{~S}$ (Fe balance). The other material is with small inclusions (designated as $S$ ) and the chemical compositions are: $1.01 \mathrm{C}, 1.45 \mathrm{Cr}, 0.35 \mathrm{Mn}$, $0.28 \mathrm{Si}, 0.015 \mathrm{P}$, and $0.01 \mathrm{~S}$ (Fe balance).

From the spheroidizing annealed bar, specimens were machined into hourglass shape with a certain amount of finishing allowance. The specimens were heated at $845^{\circ} \mathrm{C}$ for $2 \mathrm{~h}$ in vacuum, then oil-quenched and tempered for $2 \mathrm{~h}$ in vacuum at tempering temperatures of 150,180 , and $300{ }^{\circ} \mathrm{C}$, respectively. For explicitness, four groups of specimens were designated as RL180, RL300, UL150 and US150 with respect to loading mode ( $R$ : rotating bending and $\mathrm{U}$ : ultrasonic cycling), inclusion size (L: large inclusion and S: small inclusion) and tempering temperature as listed in Table 1. The geometries of two types of specimens for fatigue tests are shown in Fig. 1 . The round notch surface was ground by the grade 400, 800, 1500 and 2000 abrasive papers and polished to a smooth finish.

Before fatigue testing, tensile testing was conducted at room temperature on an MTS 810 machine with cylindrical specimens of $6 \mathrm{~mm}$ in diameter. Hardness measurement was performed by using a Vickers hardness tester at a load of $50 \mathrm{~g}$ with the load holding time of $15 \mathrm{~s}$. The mechanical properties of four groups of specimens, measured at room temperature, are listed in Table 1.

\subsection{Fatigue testing}

Fatigue testing was performed to examine the influence of inclusion location on fatigue behavior by using a "Giga-Quad" rotating bending machine at room temperature with the rotating speed of $3150 \mathrm{rpm}$ (the frequency of $52.5 \mathrm{~Hz}$ ) and the stress ratio of -1 . Fatigue testing of push-pull type was conducted to investigate the effect of inclusion size on fatigue behavior with a Shimadzu USF-2000 ultrasonic machine at a resonance frequency of $20 \mathrm{kHz}$ at room temperature, and the stress ratio of -1 .

After the fatigue testing, the fracture surfaces of fractured specimens were examined by employing a field-emission type Scanning Electron Microscope (SEM), and the chemical composition of the inclusion on fracture surface was detected by Energy Dispersive Analysis of X-ray (EDX) equipped with the SEM. With the SEM photos, the sizes of inclusions, FGA and fisheyes were measured with Image-Pro Plus (IPP) software.

\section{Experimental results}

\section{1. $S-N$ data}

Fig. 2 shows the $S-N$ data for the four groups of specimens, in which the data of Fig. 2(a) are for RL180 and RL300 specimens experienced rotary bending test and the data of Fig. 2(b) are for US150 and UL150 specimens experienced ultrasonic cycling test. The results of SEM observations on fracture surfaces show that fatigue cracks almost initiated from inclusion for the specimens with large inclusion size (RL180, RL300 and UL150), and the S-N data are divided into two categories: surface inclusion initiation and interior inclusion initiation with respect to the fatigue crack initiation site. The $S-N$ curve of RL180 specimens presents a stepwise tendency which is one of the typical characteristics of VHCF for high strength steels, while the $S-N$ data of RL300 specimens presents a duplex type. For small inclusion size specimens of US150, most fatigue crack origins initiated from interior inclusions except for a few cases which failed at low cycle fatigue regime as shown in Fig. 2(b). Furthermore, $S-N$ data of US150 and UL150 specimens experienced ultrasonic cycling show that the fatigue life has a large scatter band versus a narrow stress range with fatigue crack initiation from interior inclusions.

\subsection{Fractography}

\subsubsection{Propensity of inclusions}

Fig. 3 shows the microstructure around an inclusion on the etched surface of a specimen without fatigue testing. Martensite morphology with carbides distributed surrounding the inclusion is clearly observed. The average width of martensite lamella is measured as $378 \mathrm{~nm}$ [17]. The EDX results of the chemical compositions contained by the inclusion show that the chemical compositions of the inclusion consist of $\mathrm{Al}_{2} \mathrm{O}_{3}, \mathrm{CaO}, \mathrm{MgO}$, $\mathrm{SiO}_{2}$, etc.

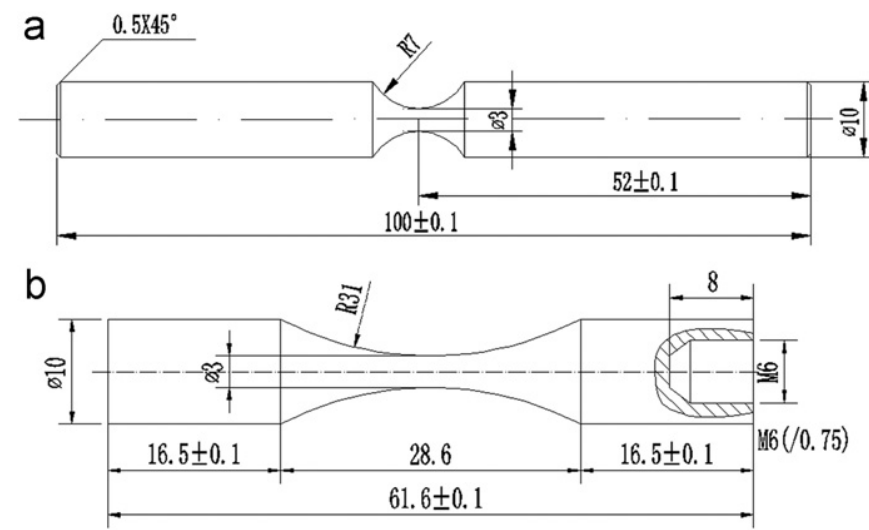

Fig. 1. Shape and dimensions (in $\mathrm{mm}$ ) of specimens for fatigue tests: (a) for rotary bending test and (b) for ultrasonic loading test.

Table 1

Properties of four groups of specimens.

\begin{tabular}{|c|c|c|c|c|c|}
\hline Specimen code & Loading mode & Inclusion size ${ }^{\mathrm{a}}(\mu \mathrm{m})$ & T.T. & $\mathrm{Hv}\left(\mathrm{kg} \mathrm{f} / \mathrm{mm}^{2}\right)$ & $\sigma_{b}(\mathrm{MPa})$ \\
\hline RL180 & $\mathrm{RB}(52.5 \mathrm{~Hz})$ & $20-60$ & $180{ }^{\circ} \mathrm{C}$ & 753 & 1849 \\
\hline RL300 & & $20-60$ & $300{ }^{\circ} \mathrm{C}$ & 666 & 1690 \\
\hline UL150 & $\mathrm{UL}(20 \mathrm{kHz})$ & $20-60$ & $150{ }^{\circ} \mathrm{C}$ & 808 & 2163 \\
\hline US150 & & $10-30$ & $150{ }^{\circ} \mathrm{C}$ & 820 & 2372 \\
\hline
\end{tabular}

a Inclusion size is measured from fatigue crack origin on fracture surfaces.

b T.T. denotes tempering temperature. 
Fig. 4 shows a pair of conjugate fracture surfaces presenting the morphology of the inclusion located in crack growth region from a specimen (RL300) subjected to rotary bending. In this region, mixed type fracture, i.e., intergranular and transgranular
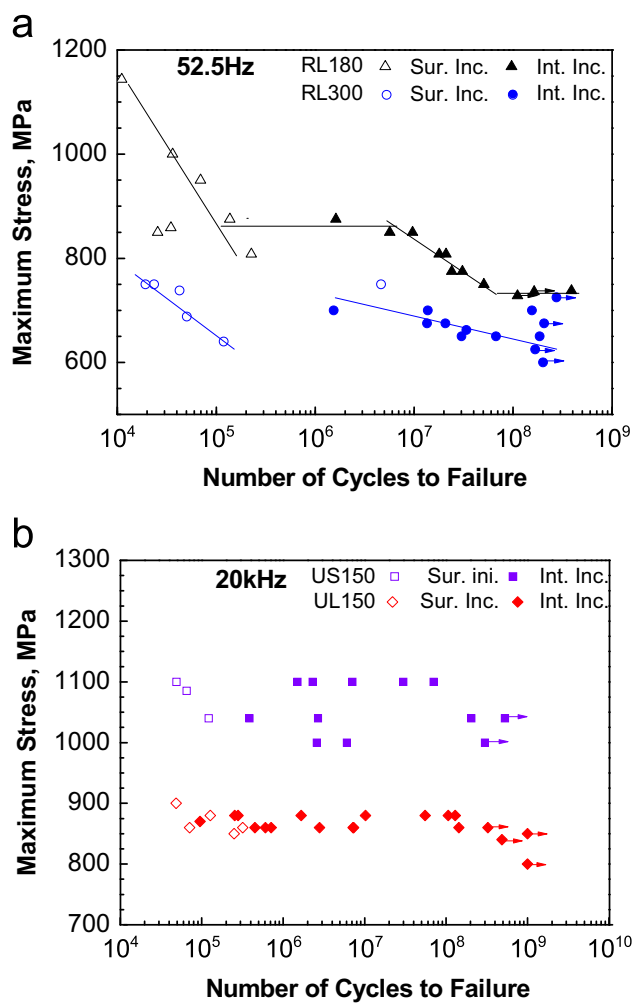

Fig. 2. $S-N$ data for RL180 and RL300 specimens experienced rotary bending test (a), and for US150 and UL150 specimens experienced ultrasonic cycling test (b). (Sur. Inc.: surface inclusion initiation; Int. Inc.: interior inclusion initiation; Sur. Ini.: surface induced initiation). cracking occurred and the inclusion was just embedded in the crack path. Such morphology of inclusion on fracture surface is distinguished from that of the inclusion as fatigue crack origin. Usually there is a ring of wedge-shape crack around the inclusion in the plane perpendicular to fracture surface. And the wedgeshape crack probably formed during the cold-drawing process.

\subsubsection{Inclusion location and fracture patterns}

For convenience, the inclusions as fatigue crack origins are classified into three types according to their locations: surface inclusion, subsurface inclusion and interior inclusion, which is defined by the inclusion depth $\left(d_{\text {inc }}\right)$ with respect to specimen surface. In this paper, subsurface inclusions are those with $d_{\text {inc }}$ between 5 and $40 \mu \mathrm{m}$, and interior inclusions are those with $d_{\mathrm{inc}}$ large than $40 \mu \mathrm{m}$. Surface inclusions are contacted with specimen surface and $d_{\text {inc }}$ is less than $5 \mu \mathrm{m}$.

Based on the results of fracture surface observations, the fatigue fracture patterns due to rotary bending test are classified into four types: Pattern I: "Surface fisheye" as shown in Fig. 5, Pattern II: "Interior fisheye" as shown in Fig. 6, Pattern III "FGA+Surface fisheye" as shown in Fig. 7, and Pattern IV "FGA+Interior fisheye" as shown in Fig. 8. As shown in Figs. 5 and 6 , the large inclusion at surface or in subsurface induces fatigue crack initiation and early growth, which leads to a shorter fatigue life. The surface or interior fisheye region indicates the process of fatigue crack early growth. When the inclusion is located in the interior of specimen, both surface fisheye and interior fisheye are observed with FGA around the inclusion as shown in Figs. 7 and 8, and the inclusion is much closer to the specimen surface in Fig. 7, for which the fracture pattern is "FGA+Surface fisheye".

Pattern IV (Fig. 8) is a complete type of VHCF failure both under rotary bending test and push-pull type of ultrasonic cycling test. However, Pattern III induced by subsurface inclusion within VHCF regime is only observed under rotary bending test, which is rarely reported in VHCF investigations.
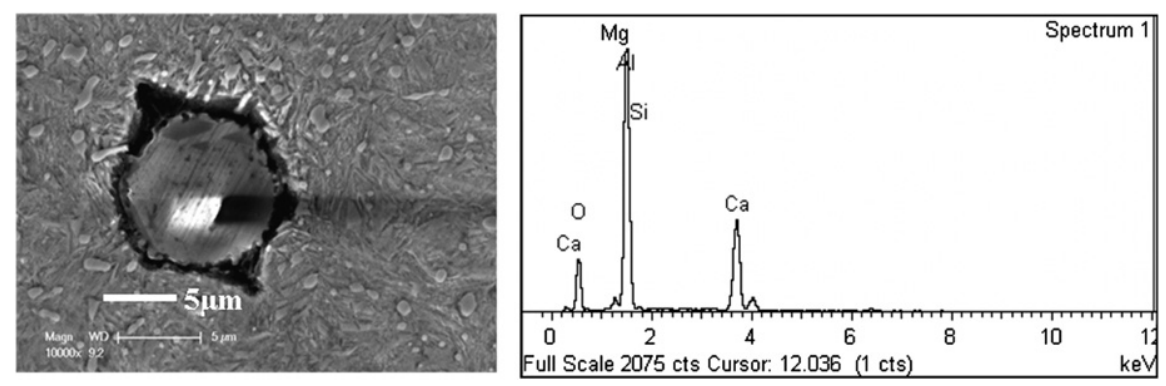

Fig. 3. SEM image showing an inclusion with surrounding microstructure on etched surface and its chemical compositions by EDX examination.
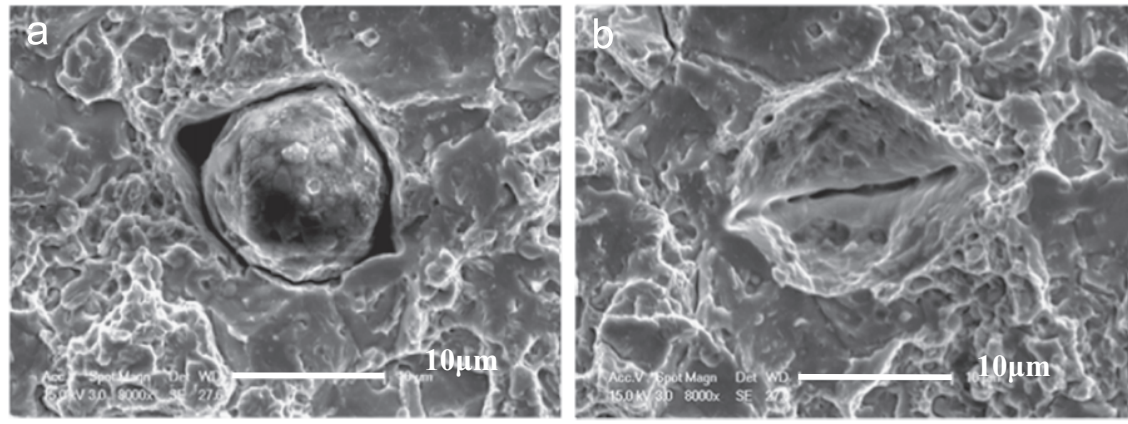

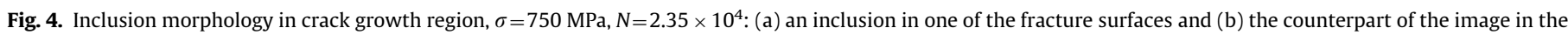
conjugate fracture surface. 


\subsubsection{Inclusion size and fatigue crack initiation}

Fig. 9 shows an example of fatigue crack originated from an interior large inclusion instead of a comparatively small inclusion at the specimen surface although the stress was higher for the surface location, indicating large inclusion in preference to small one to cause fatigue crack initiation. The size of the large inclusion at the subsurface is $29 \mu \mathrm{m}$ and that of the small one at the specimen surface is $11 \mu \mathrm{m}$.

The push-pull type of ultrasonic cycling test makes the stress distribute uniformly in the same cross section, and VHCF crack often originates from interior inclusion for which the influence of inclusion depth may be ignored. Fig. 10 is another example of fatigue crack initiation from a larger inclusion in VHCF regime under ultrasonic cycling test. It is noticed that there is no obvious trace of damage for the smaller inclusion on the fracture surface. The values of stress intensity factor of the two inclusions are 3.86

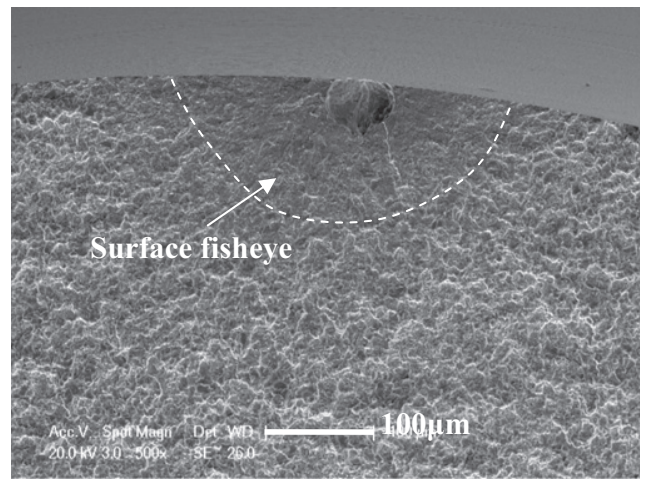

Fig. 5. Pattern I: surface fisheye from the surface inclusion $\left(d_{\mathrm{inc}}=0, \sigma=875 \mathrm{MPa}\right.$ $N=1.37 \times 10^{5}$ )

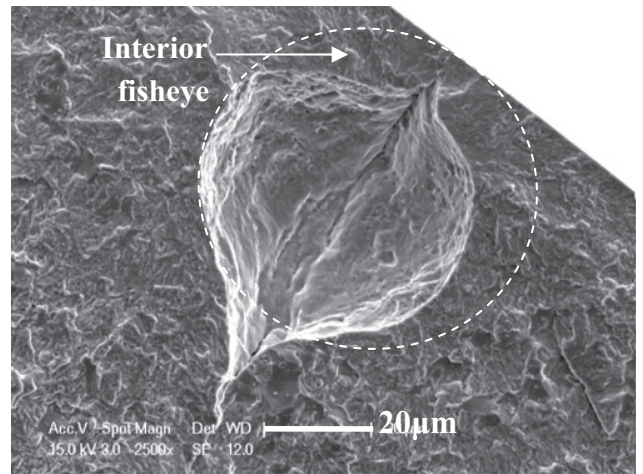

Fig. 6. Pattern II: interior fisheye from subsurface large inclusion $\left(d_{\text {inc }}=6 \mu \mathrm{m}\right.$ $\sigma=850 \mathrm{MPa}, N=2.57 \times 10^{4}$ ). and $3.65 \mathrm{MPa} \mathrm{m}^{1 / 2}$, respectively, and the fatigue crack started from the bigger one ,with a little higher value of stress intensity factor. It is confirmed that inclusion size is one of important factors dominating the process of VHCF crack initiation.

\section{Discussion}

\subsection{Effect of inclusion location on fracture pattern transition}

The value of $\sqrt{\text { area }}$ is used to characterized the size of inclusion, FGA or fisheye on fracture surface. Thus we are able to calculate the stress intensity factor range at the edge of interior inclusion, FGA or fisheye with the following equation [18]:

$\Delta K=0.5 \sigma \sqrt{\pi \sqrt{\text { area }}}$

For surface inclusion and fisheye, the coefficient of 0.5 is replaced by 0.65 in the calculation.

Fig. 11 shows the influence of inclusion location on fracture pattern transition under rotary bending test. The correlation between $d_{\text {inc }}$ and number of cycles to failure with regard to fracture patterns is shown in Fig. 11(a). The values of $d_{\text {inc }}$ ranged from 0 to $200 \mu \mathrm{m}$, and the fatigue life presents an increase tendency with the increase of $d_{\text {inc }}$. Most data of fatigue life are within $2 \times 10^{5}$ cycles with the maximum value of $4.63 \times 10^{6}$ for surface inclusion induced failures (Pattern I) and interior inclusions lead to VHCF failures (Pattern IV). While high cycle fatigue and VHCF occurred at the same time for subsurface inclusions with Patterns II and III, the inclusion size may play a great role in transition between these two patterns.

Fig. 12 illustrates the results of stress intensity factor range for inclusion, FGA and fisheye as a function of fatigue life. The value of $\Delta K_{\text {inc }}$ tends to decrease slightly as fatigue life increases, while $\Delta K_{\mathrm{FGA}}$ keeps almost a constant with an average of 5.34 and 4.34 MPa m ${ }^{1 / 2}$ for RL180 and RL300, respectively, as shown in Fig. 12(a). Based on the conclusion that $\Delta K_{\mathrm{FGA}}$ is close to the traditional crack growth threshold $\Delta K_{\text {th }}$ [17], four fracture patterns are summarized in relation to the value of $d_{\text {inc }}$ and $\Delta K_{\text {inc }}$ :

Pattern I Surface Fisheye : $\Delta K_{\text {inc }} \geq \Delta K_{\text {th }}, \quad d_{\text {inc }} \approx 0 \mu m$

Pattern II Interior Fisheye : $\Delta K_{\text {inc }} \geq \Delta K_{\text {th }}, \quad 5 \mu m \leq d_{\text {inc }}<40 \mu m$

Pattern III FGA+Surface Fisheye : $\Delta K_{\text {inc }}<\Delta K_{\text {th }}, \quad 5 \mu m \leq d_{\text {inc }}<40 \mu m$

Pattern IV FGA+Interior Fisheye : $\Delta K_{\text {inc }}<\Delta K_{\text {th }}, \quad 40 \mu m \leq d_{\text {inc }} \leq 200 \mu m$

In Eq. (2), $\Delta K_{\mathrm{th}}$ is taken as the value of $\Delta K_{\mathrm{FGA}}$ for RL180 and RL300. It is seen that the value of $\Delta K_{\mathrm{inc}}$ is always lower than $\Delta K_{\mathrm{th}}$ for the specimens fractured with FGA.

Note that the value of $\Delta K_{\mathrm{FGA}}$ for a RL180 specimen fractured with surface fisheye pattern is $4.40 \mathrm{MPa} \mathrm{m}{ }^{1 / 2}$ which is obviously lower than the common value between 5 and $6 \mathrm{MPa} \mathrm{m}^{1 / 2}$ for the other RL180 specimens fractured with interior fisheye pattern.
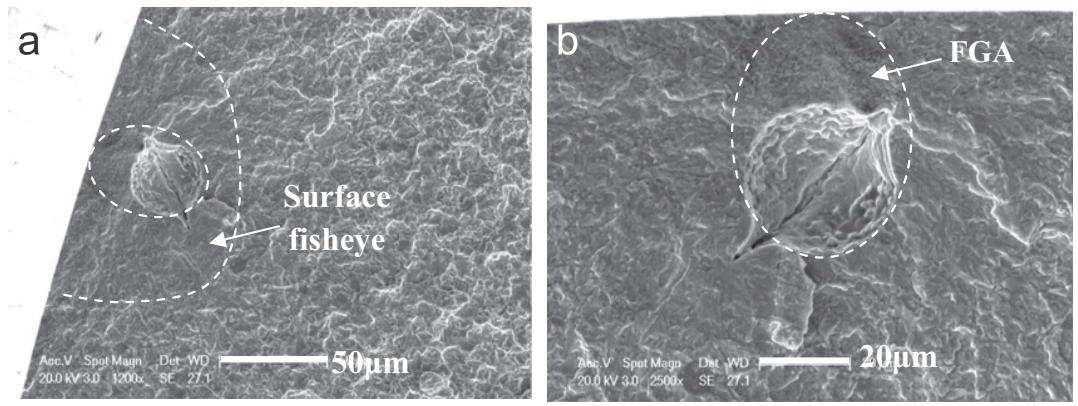

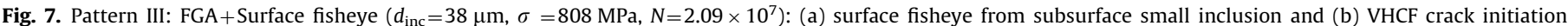
with FGA. 

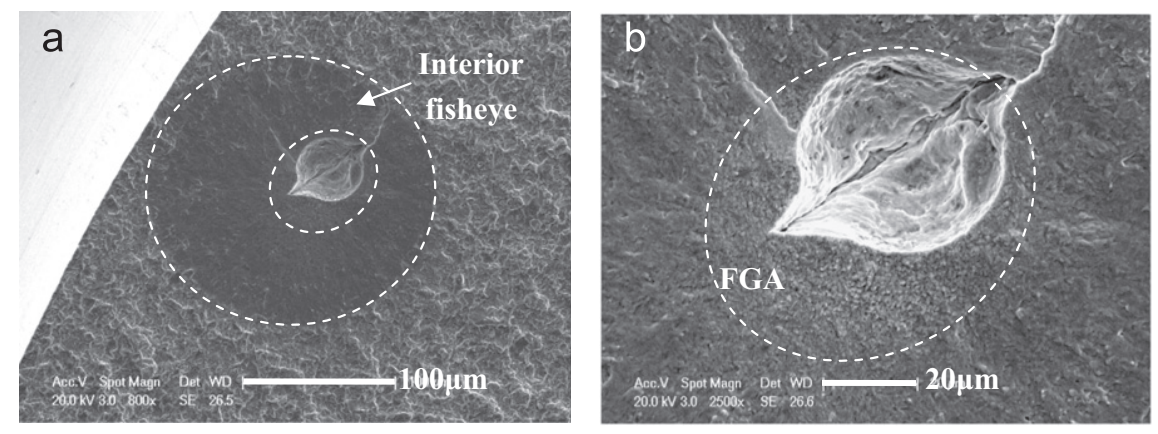

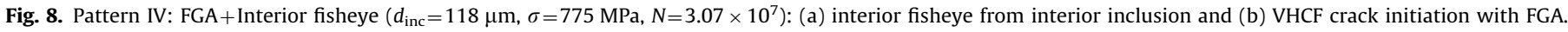

a

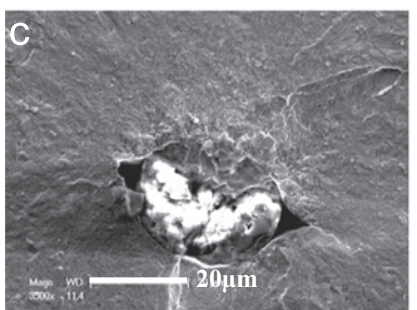

d

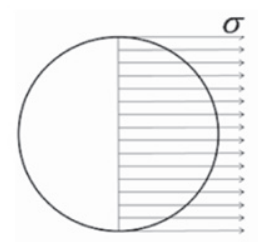

Fig. 10. Ultrasonic cycling test ( $\sigma=860 \mathrm{MPa}, N=2.73 \times 10^{7}$ ), VHCF crack initiation from the bigger inclusion: (a) two inclusions distributed on fracture surface, (b) the relatively smaller inclusion in crack propagation region with $\sqrt{\text { area }_{\text {inc }}}=$ $23 \mu \mathrm{m}$, (c) the bigger inclusion as crack origin with $\sqrt{\operatorname{area} a_{\mathrm{inc}}}=26 \mu \mathrm{m}$, and (d) stress distribution on cross section.

Note also that the values of $\Delta K_{\text {fisheye }}$ keep a constant for both interior fisheye pattern and surface fisheye pattern of each group of specimens as shown in Fig. 12(b), and the sizes of interior fisheyes depend on the location of the inclusion with a fit of $y=0.76 x$ (Fig. 11(b)).

These results reveal that the formation of FGA and interior fisheye was terminated once the FGA or interior fisheye grew up to reach the specimen surface although the value of $\Delta K_{\mathrm{FGA}}$ or $\Delta K_{\text {fisheye }}$ was lower than the normal critical value. Otherwise, interior fisheye occurred from interior inclusion with $\Delta K_{\mathrm{FGA}}$ corresponding to the critical value of $\Delta K_{\mathrm{th}}$. For a specimen at the given stress level, where fatigue cracks originate is not only related with the inclusion location but also related with other factors, e.g., inclusion size (Figs. 9 and 10), hence it is the location of the inclusion as crack origin that makes the transition of fracture pattern.
C

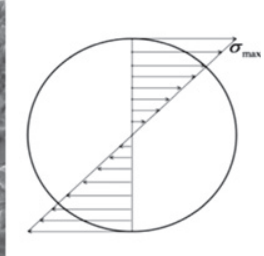

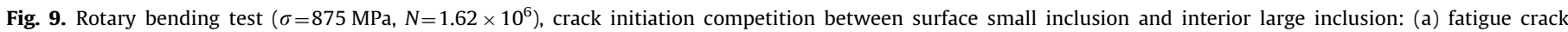
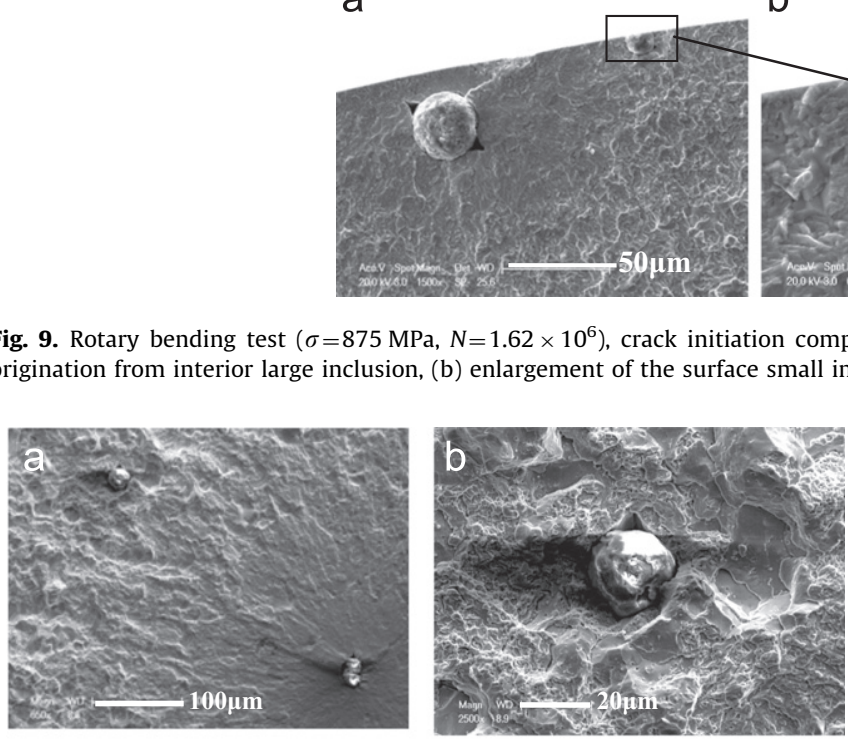

b

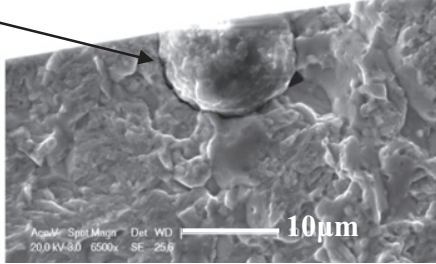



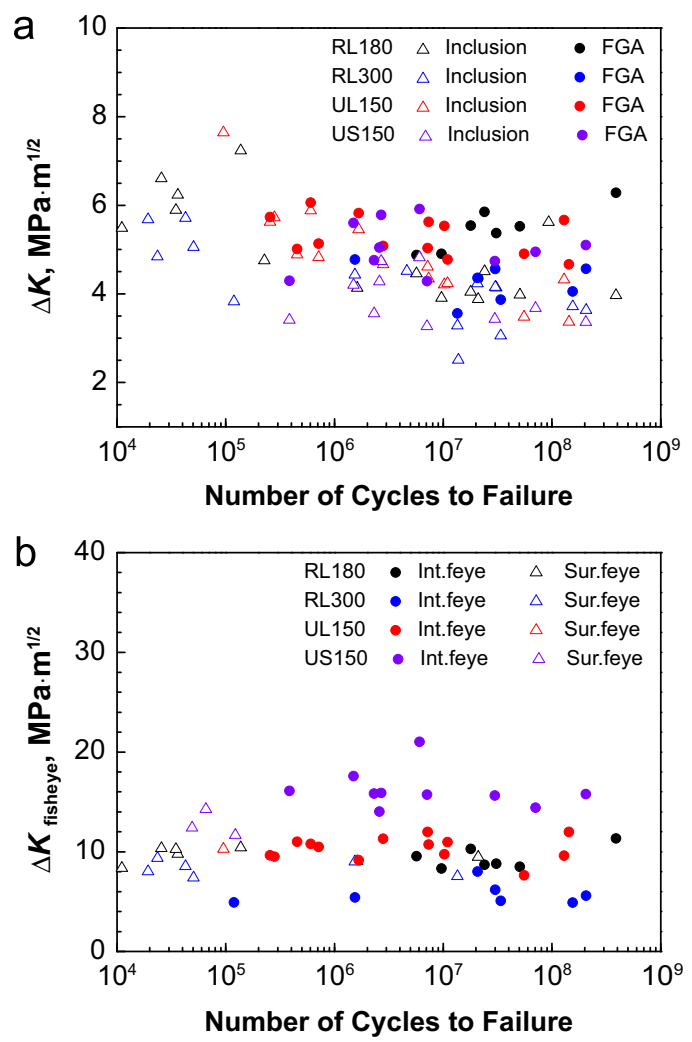

Fig. 12. (a) $\triangle K$ of FGA or inclusion vs number of cycles to failure and (b) $\triangle K_{\text {fisheye }}$ vs number of cycles to failure (Int. feye: interior fisheye; Sur. feye: surface fisheye).

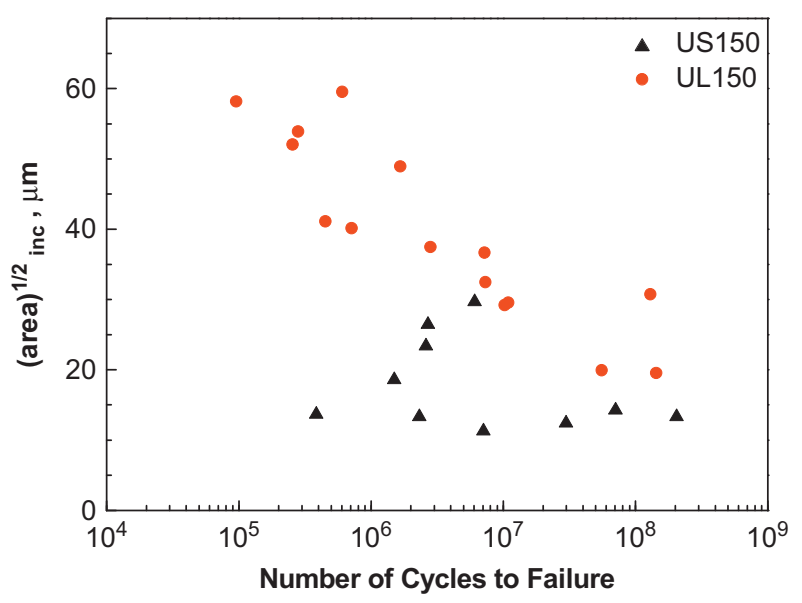

Fig. 13. Influence of inclusion size on fatigue life in terms of $\sqrt{a r e a_{\text {inc }}}$.

applied load. As shown in Fig. 13, the sizes of inclusions as fatigue crack origin on fracture surfaces are determined in terms of $\sqrt{\text { area }}$ with IPP software. It is seen that the average inclusion size of large inclusion specimens UL150 reaches $40 \mu \mathrm{m}$ with a uniform distribution from 20 to $60 \mu \mathrm{m}$, and the average inclusion size of small inclusion specimens US150 is about $17 \mu \mathrm{m}$ with a range from 10 to $30 \mu \mathrm{m}$.

FGA and interior fisheye were often observed for Pattern IV mentioned above for most specimens of UL150 and US150. For UL150, the stress was controlled at two close levels of 860 and $880 \mathrm{MPa}$ (Fig. 2(b)), and the fatigue life decreased from $10^{8}$ to $10^{5}$ cycles when the inclusion size increased from 20 to $60 \mu \mathrm{m}$
(Fig. 13), suggesting that large inclusions induced an early initiation of fatigue crack and led to a shorter fatigue life, whereas relatively small inclusions upgraded the fatigue life with FGA formation.

It is noted that ratio of fatigue limit to tensile strength is used to reflect the fatigue strength change for specimens with different tensile strength, and this ratio tends to decrease as the tensile strength increases for this type of material [6]. In this study, the ratio of $\sigma_{\max }$ to $\sigma_{\mathrm{b}}$ is about 0.45 for US150, while it is about 0.40 for UL150 which has lower tensile strength. The present results reveal that this tendency is reversed by US150 with relatively smaller inclusions, and the fatigue strength has been improved about $12.5 \%$ by decreasing the inclusion size of the material in terms of $\sigma_{\max } / \sigma_{\mathrm{b}}$ ratio.

Fig. 14 shows the tendency of large inclusions decreasing fatigue life and fatigue strength. The relationship between the ratio of $\sqrt{\operatorname{area}_{\mathrm{FGA}}} / \sqrt{\operatorname{area}_{\text {inc }}}$ and fatigue life is plotted in Fig. 14(a), which shows that the large size of FGA gives rise to the longer fatigue life for both UL150 and US150 specimens. As we know, the formation of FGA corresponds to the period of VHCF crack initiation, which consumes the majority of the fatigue life, and $\Delta K_{\mathrm{FGA}}$ tends to be a constant value of $\Delta K_{\mathrm{th}}$. On one hand, the specimens with larger inclusions result a smaller ratio of $\sqrt{\operatorname{area}_{\mathrm{FGA}}} / \sqrt{\operatorname{area}_{\text {inc }}}$ at a given stress level, leading to a shorter failure cycles because of the smaller value of net FGA (area of the inclusion is subtracted). On the other hand, the stress level required for FGA formation will be lowered when the inclusion size is larger than the critical size determined by the maximum threshold value of the material $\Delta K_{\text {th,max }}$, or the fatigue crack will enter into the early growth period without FGA formation as shown in Fig. 14(b).
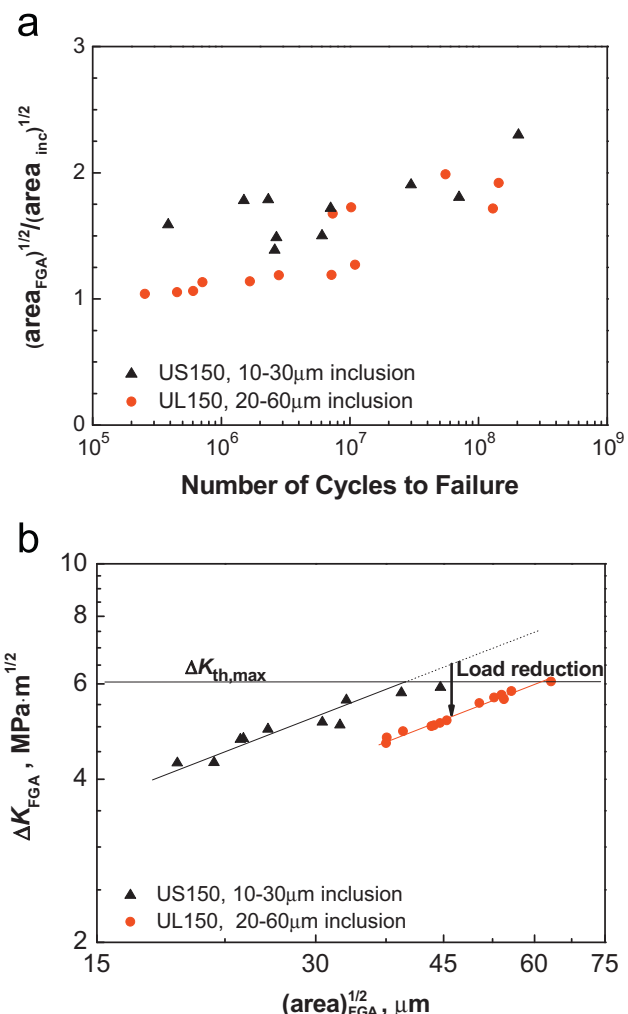

Fig. 14. Tendency of large inclusions decreasing fatigue life and strength: (a) increasing trend of $\sqrt{\operatorname{area}_{\mathrm{FGA}}} / \sqrt{\operatorname{area}_{\mathrm{inc}}}$ with fatigue life and (b) reducing load to reach the conditions of FGA formation for specimens with large inclusions. 


\subsection{Mechanism of FGA formation}

Based on the model of "polygonization and debonding" proposed by Sakai [11], recently it is further proposed that FGA formed when the martensite lath was segmented into fine packets and the dislocation cells formed, with the help of stress concentration caused by the inclusion during the cycling process by Li et al. [19]. It is also noticed that, the studies on the threshold for FGA formation by Zhao et al. [17] revealed that the plastic zone size of FGA crack was close to the width of martensite, and got the further deduction that the size of FGA finished when its plastic zone was beyond the martensite width. It is believed that martensite dimension plays an important role in the formation of FGA. Thus in the view of martensite segmentation during FGA formation, it is suggested that FGA has a certain thickness in its vertical direction.

The fact is that the closer to the inclusion or the longer fatigue cycles yields a clearer FGA, and even sometimes one can hardly draw a line between FGA and fisheye. One may consider that the different degrees of granulated FGA leading to the result, thus the concept of the thickness for the fine granular layer $h$ is proposed to represent the degree of granulated FGA. In this way, there are two characteristic dimensions of FGA: $\sqrt{\operatorname{area}_{\mathrm{FGA}}}$ and $h$ as shown in Fig. 15(a). In addition to the parameter of $\sqrt{\operatorname{area}_{\mathrm{FGA}}}$ which can be predicted by the threshold value of crack growth for the material at a given stress level, the value of $h$ is about one or several times of the fine granular size depending on the fatigue cycles during the period of its formation, and this value decreases as FGA grows up with the decline of the effective stress cycles for FGA formation. Thus a clearer FGA often presented in the region closer to the inclusion and in the fracture surface enduring longer fatigue cycles as Figs. 15(b) and (c) show.

\subsection{Mean crack growth rate in FGA}

In the discussion of the influence of inclusion location, we have mentioned that the surface fisheye was observed around FGA for rotating bending specimens. Thus the fatigue life consumed in FGA can be calculated if the fatigue life of surface fisheye can be estimated.

By comparison of the value of $\Delta K_{\text {inc }}$ with $\Delta K_{\mathrm{FGA}}$ for each specimen group as shown in Fig. 12(a), it is seen that the value of $\Delta K_{\text {inc }}$ is greater than or equal to the value of $\Delta K_{\mathrm{FGA}}$ for surface inclusion induced failures. And it is assumed that fatigue crack propagation is dominant during the formation of surface fisheye for this type of failure. Thus the mean crack propagation rate in surface fisheye is calculated for all the specimens fractured with Pattern I by rotating bending test with the following equation:

$$
\overline{\left(\frac{\mathrm{d} a}{\mathrm{~d} N}\right)_{\text {fisheye }, \mathrm{s}}}=\frac{\sqrt{\operatorname{area}_{\text {fisheye }, \mathrm{s}}}}{N}
$$

In Eq. (3), the fatigue life due to the crack propagation outside fisheye is neglected. The calculation results are plotted in Fig. 16. Meanwhile this calculated values are compared with the experimental results of stable crack growth rate with the similar material done by Shiozawa et al. [20]. It is seen that the mean

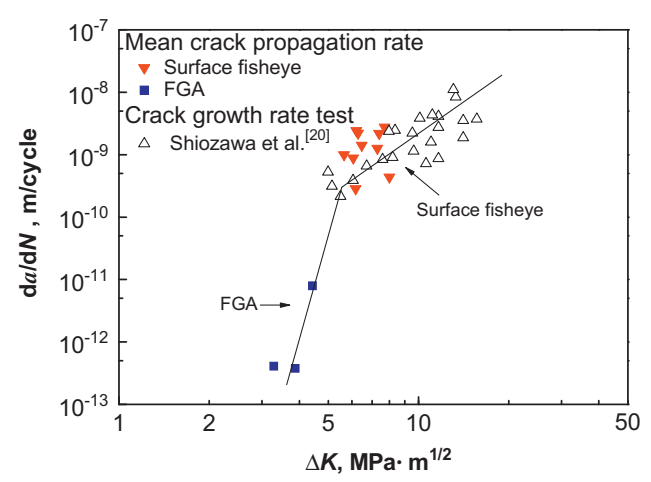

Fig. 16. Evaluation of the mean crack growth rate in FGA.
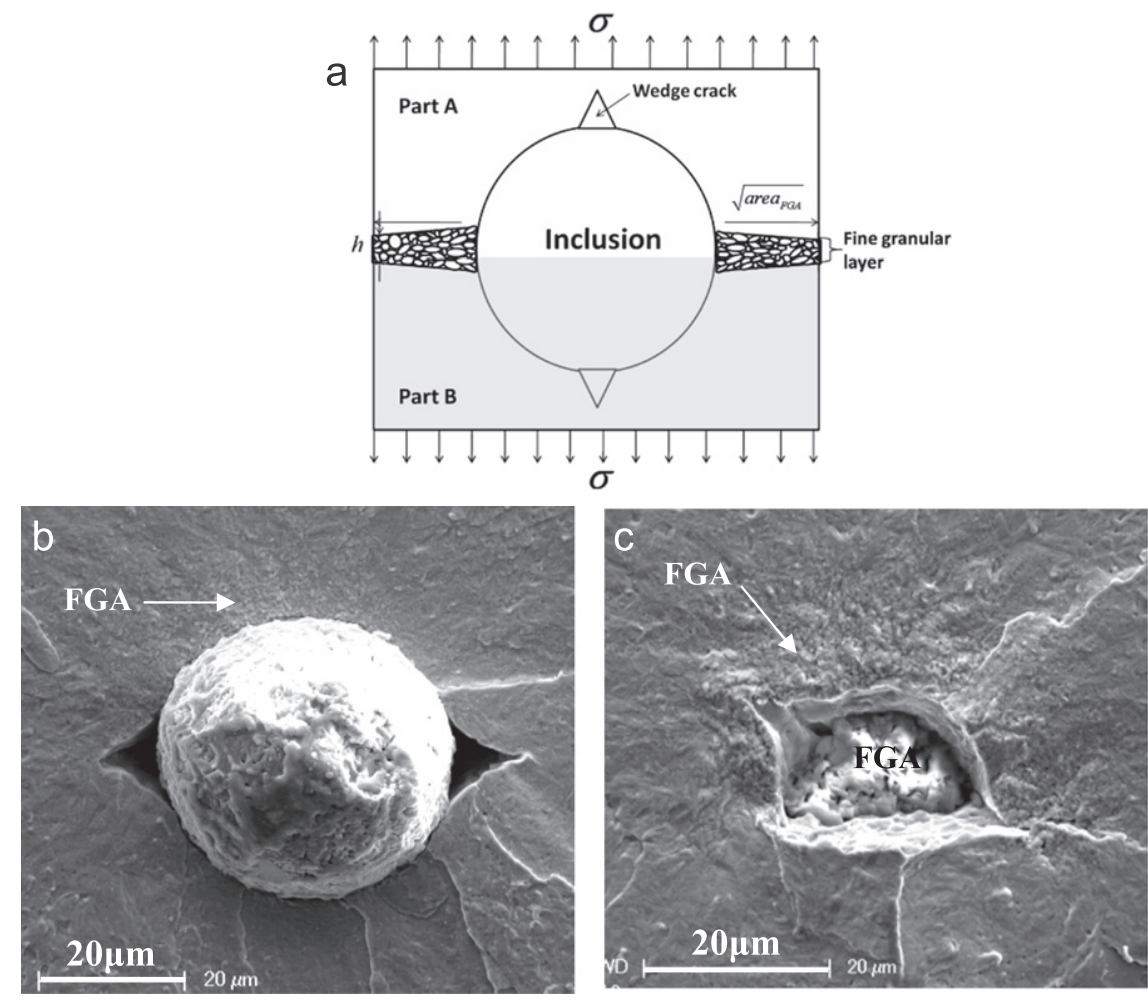

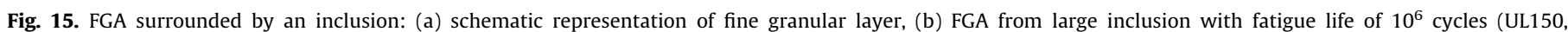
$\sigma=860 \mathrm{MPa}, N=2.81 \times 10^{6}$ ), and (c) clearer FGA from smaller inclusion with longer fatigue life of $10^{8}$ cycles $\left(\mathrm{UL150}, \sigma=860 \mathrm{MPa}, N=1.43 \times 10^{8}\right.$ ). 
Table 2

Crack data for specimens subjected to rotating bending and fractured with Pattern III: FGA+surface fisheye.

\begin{tabular}{|c|c|c|c|c|c|c|c|c|}
\hline$\sigma_{\max }(\mathrm{MPa})$ & $N$ (cycles) & $d_{\text {inc }}(\mu \mathrm{m})$ & $2 a_{\text {Inc }}(\mu \mathrm{m})$ & $2 a_{\mathrm{FGA}}(\mu \mathrm{m})$ & $2 a_{\text {fisheye }}(\mu \mathrm{m})$ & $N_{p}$ (cycles) & $N_{\mathrm{FGA}}($ cycles $)$ & $(\mathrm{d} a / \mathrm{d} N)_{\mathrm{FGA}}(\mathrm{m} /$ cycle $)$ \\
\hline 808 & $2.09 \times 10^{7}$ & 38 & 30 & 38 & 108 & $4.56 \times 10^{5}$ & $2.04 \times 10^{7}$ & $3.77 \times 10^{-13}$ \\
\hline 675 & $1.35 \times 10^{7}$ & 8 & 30 & 36 & 98 & $8.22 \times 10^{5}$ & $1.27 \times 10^{7}$ & $4.09 \times 10^{-13}$ \\
\hline 700 & $1.55 \times 10^{6}$ & 6 & 51 & 60 & 76 & $5.23 \times 10^{5}$ & $1.03 \times 10^{6}$ & $7.96 \times 10^{-12}$ \\
\hline
\end{tabular}

crack propagation rate is at the same level of the stable crack growth rate, thus the fatigue crack propagation behavior within surface fisheye for Pattern I can be described with Paris relation fitted by experimental results:

$\mathrm{d} a / \mathrm{d} N=1.03 \times 10^{-12} \Delta K^{3.28}$

Table 2 lists the data of specimens fractured with Pattern III. Since the fatigue cycles of crack propagation in surface fisheye $N_{\mathrm{p}}$ can be estimated by Paris relation, the fatigue crack initiation life consumed in FGA $N_{\mathrm{FGA}}$ is obtained as $\left(N-N_{\mathrm{p}}\right)$. And we get the mean crack growth rate in FGA:

$\overline{\left(\frac{\mathrm{d} a}{\mathrm{~d} N}\right)_{\mathrm{FGA}}}=\frac{\sqrt{\mathrm{area}_{\mathrm{FGA}}}-\sqrt{\mathrm{area}_{\mathrm{inc}}}}{N-N_{p}}$

In Eq. (5), $N_{p}$ is calculated with Paris relation of Eq. (4), and the calculation method is similar with that in Ref. [21]. In this way, $(\mathrm{d} a / \mathrm{d} N)_{\mathrm{FGA}}$ is of the magnitude of $10^{-13} \mathrm{~m} /$ cycle for fatigue cycles at $10^{7}$ cycles, and of the magnitude of $10^{-12} \mathrm{~m} /$ cycle for fatigue cycles at $10^{6}$ cycles. The main reason for the increase of crack growth rate in FGA is the reduction of net FGA area caused by a larger inclusion. Other studies on crack propagation rate show that crack propagation rate in FGA is close to $10^{-12} \mathrm{~m} /$ cycle obtained by Sun et al. [4] and Stanzl-Tschegg et al. [22]. The mean crack growth rates in FGA are also plotted in Fig. 16. It is seen that below (FGA) and above (surface fisheye) the threshold, the crack propagation rate differs definitely. Such a low rate of FGA crack, i.e., FGA formation is attributed to the energy accumulation of dislocation movement for VHCF crack initiation in FGA [21].

\section{Conclusions}

The following conclusions are drawn for the high strength steels subjected to rotary bending test at $52.5 \mathrm{~Hz}$ and ultrasonic cycling test at $20 \mathrm{kHz}$.

The location of inclusion has significant influence on fatigue crack initiation and early growth for the specimens under rotary bending test. The fracture morphology changes as the increase of the inclusion depth away from specimen surface. Four fracture patterns exist for the high strength steels, i.e., Surface fisheye, Interior fisheye, FGA + Surface fisheye, and FGA+Interior fisheye.

The material with smaller inclusions has higher fatigue strength and a longer fatigue life. Under the same loading condition, the fatigue life is prolonged by two to three orders of magnitude with reducing the inclusion size by about one third.
Large inclusions lower the proportion of net FGA at given stress level, leading to a shorter VHCF life. VHCF strength is degraded with decreasing critical stress of FGA formation for large inclusions.

The fracture pattern of "FGA + surface fisheye" provides a way to estimate the crack growth rate in FGA without concerning the fatigue life consumed by interior fisheye which is difficult to be measured. The mean crack growth rate in FGA formed in VHCF regime is at the magnitude of $10^{-13} \mathrm{~m} /$ cycle.

\section{Acknowledgements}

The authors gratefully acknowledge the support of the National Natural Science Foundation of China (Grant Nos. 11021262 and 11172304), and the National Basic Research Program of China (Grant No. 2012CB937500).

\section{References}

[1] T. Naito, H. Ueda, M. Kikuchi, Metall. Trans. A 15A (1984) 1431-1436.

[2] Y. Nakamura, T. Sakai, H. Hirano, C. Ravi, Int. J. Fatigue 32 (2010) 621-626.

[3] Y. Hong, A. Zhao, G. Qian, Acta Metall. Sinica 45 (2009) 769-780.

[4] C. Sun, J. Xie, A. Zhao, Z. Lei, Y. Hong, Fatigue Fract. Eng. Mater. Struct. 35 (2012) 638-647.

[5] G. Qian, C. Zhou, Y. Hong, Acta Mater. 59 (2011) 1321-1327.

[6] A. Zhao, J. Xie, C. Sun, Z. Lei, Y. Hong, Int. J. Fatigue 38 (2012) 46-56

[7] Y. Hong, A. Zhao, G. Qian, C. Zhou, Metall. Mater. Trans. A 43A (2012) $2753-2762$.

[8] Z. Duan, H.J. Shi, X.F. Ma, Fatigue Fract. Eng. Mater. Struct. 34 (2011) 832-837.

[9] Y. Murakami, T. Nomoto, T. Ueda, Y. Murakami, Fatigue Fract. Eng. Mater. Struct. 23 (2000) 893-902.

[10] K. Shiozawa, Y. Morii, S. Nishino, L.T. Lu, Int. J. Fatigue 28 (2006) 1521-1532.

[11] T. Sakai, J. Solid Mech. Mater. Eng. 3 (2007) 425-439.

[12] Y. Murakami, M. Takada, T. Toriyama, Int. J. Fatigue 20 (1998) 661-667.

[13] K. Shiozawa, L.T. Lu, Fatigue Fract. Eng. Mater. Struct. 25 (2002) 813-822.

[14] Y. Murakami, T. Nomoto, T. Ueda, Fatigue Fract. Eng. Mater. Struct. 22 (1999) 581-590.

[15] M.D. Chapetti, T. Tagawa, T. Miyata, Mater. Sci. Eng. A 356 (2003) 236-244.

[16] J.M. Zhang, S.X. Li, Z.G. Yang, G.Y. Li, W.J. Hui, Y.Q. Weng, Int. J. Fatigue 29 (2007) 765-771.

[17] A. Zhao, J. Xie, C. Sun, Z. Lei, Y. Hong, Mater. Sci. Eng. A 528 (2011) 6872-6877.

[18] Y. Murakami, S. Kodama, S. Konuma, Trans. Jpn. Soc. Mech. Eng. A 54 (1988) 688-696.

[19] W. Li, T. Sakai, Q. Li, L.T. Lu, Int. J. Fatigue 32 (2010) 1096-1107.

[20] K. Shiozawa, L.T. Lu, S. Ishihara, Fatigue Fract. Eng. Mater. Struct. 24 (2001) 781-790.

[21] Q.Y. Wang, C. Bathias, N. Kawagoishi, Q. Chen, Int. J. Fatigue 24 (2002) 1269-1274.

[22] S. Stanzl-Tschegg, B. Schonbauer, Procedia Eng. 2 (2010) 1547-1555. 